\title{
Teor de umidade e densidade básica da madeira de nove espécies comerciais amazônicas
}

\author{
Lívia Helena Carrera SILVEIRA ${ }^{1}$, Alba Valéria REZENDE ${ }^{1}$, Ailton Teixeira do VALE ${ }^{1}$
}

RESUMO

Este trabalho teve como objetivo agrupar espécies com características de teor de umidade e densidade básica da madeira semelhantes para compor cargas mistas de espécies para secagem em câmaras industriais de secagem da madeira. Foram amostradas nove espécies em floresta de terra firme na Amazônia Central com três repetiçóes por espécie. Em todas as espécies os teores de umidade não diferiram entre base e topo do tronco, o que confere estabilidade da madeira durante o processo de secagem. Os valores de densidade básica variaram de 0,561 a $0,904 \mathrm{~g} \mathrm{~cm}^{-3}$. A partir do presente estudo foi possível separar as espécies em três grupos com base na densidade básica e teor de umidade, o primeiro com as espécies Minquartia guianensis, Lecythis poiteaui, Mezilaurus itauba, Manilkara huberi e Brosimum rubescens que são consideradas madeiras pesadas (densidade variando de 0,835 a $0,904 \mathrm{~g} \mathrm{~cm}^{-3}$ ), de secagem mais lenta ou difícil. O segundo grupo contem as espécies Clarisia racemosa e Ocotea rubra (densidade média de 0,665 e $0,720 \mathrm{~g} \mathrm{~cm}^{-3}$, respectivamente) e o terceiro grupo com as espécies Parkia paraensis e Brasimum parinarioides que apresentam densidade média e secagem rápida (densidade de $0,561 \mathrm{e} 0,588 \mathrm{~g} \mathrm{~cm}^{-3}$, respectivamente).

PALAVRAS-CHAVE: grupo de espécies florestais, secagem em estufa, densidade da madeira.

\section{Moisture content and basic wood density of nine commercial Amazonian tree species}

\section{ABSTRACT}

The aim of this work was to group species with similar moisture content and basic wood density in order to compose mixed species loads for industrial kiln drying. Nine tree species with three repetitions each were sampled at an Upland Forest in Central Amazonia. For all the species the moisture content was the same for top and base portions of the trunk, which gives stability during wood drying processes. The basic wood density values varied from 0,561 to $0,904 \mathrm{~g} \mathrm{~cm}^{-3}$. The species were divided in three groups based on basic density and moisture content; the first one included the species Minquartia guianensis, Lecythis poiteaui, Mezilaurus itauba, Manilkara huberi and Brosimum rubescens which are considered dificult or slow drying heavy woods (density ranging between 0,835 to $0,904 \mathrm{~g} \mathrm{~cm}^{-3}$ ). The second group was composed with the species Clarisia racemosa and Ocotea rubra (avarage density of 0,665 and $0,720 \mathrm{~g} \mathrm{~cm}^{-3}$, respectively), the third group includes Parkia paraensis and Brasimum parinarioides that showed average density and were considered fast drying woods (density of 0,561 e $0,588 \mathrm{~g}$ $\mathrm{cm}^{-3}$, respectively).

KEYWORDS: group of forest species, kiln drying, wood density. 


\section{INTRODUÇÃO}

A secagem da madeira é um processo importante para garantir a qualidade do produto madeireiro. A secagem correta proporciona melhoria das características de trabalhabilidade, redução da movimentação dimensional, do ataque por fungos e insetos e maior controle de defeitos. Em geral, as propriedades físicas e mecânicas dependem do teor de umidade da madeira (Logsdon e Calil Junior 2002; Glass e Zelinka 2010).

A secagem artificial de madeiras da floresta Amazônica é de difícil gerenciamento por causa da grande quantidade de espécies exploradas, as espécies madeireiras exploradas comercialmente podem passar de 70 . A variabilidade dessas espécies é muito grande e a densidade pode variar de 0,34 a $0,97 \mathrm{~g} \mathrm{~cm}^{-3}$ (Nascimento et al. 1997).

Para tentar contornar esta situação, é necessário agrupar espécies com as mesmas características para compor cargas que preencham todo o volume da câmara de secagem industrial. $\mathrm{O}$ agrupamento das espécies é feito a partir de várias características como densidade básica, teor de umidade inicial, permeabilidade da madeira a fluidos, retraçáo transversal e radial, grâ, textura e aspectos anatômicos da madeira (Andrade et al. 2001; Marques 2008). Porém as características mais facilmente obtidas e mais utilizadas são densidade básica e teor de umidade inicial (Simpson e Baah 1989; Simpson e Verrill 1997).

O teor de umidade é inversamente proporcional à densidade da madeira, ou seja, quanto maior a quantidade de água, menor a quantidade dos outros elementos químicos da madeira - celulose, hemicelulose e lignina (Foelkel et al. 1971).

A densidade básica é um importante parâmetro para avaliação da qualidade da madeira, sendo uma variável complexa, pois resulta da combinação de diversos fatores como dimensão das fibras, espessura da parede celular, volume dos vasos e parênquimas, proporçâo entre madeira do cerne e alburno e arranjo dos elementos anatômicos (Foelkel $e t$ al. 1971).

A densidade básica pode ser determinada pela razão entre a massa seca e o volume saturado da madeira ou pelo método de máximo teor de umidade, em pequenas amostras. Este último método é simples e requer apenas a determinação da massa saturada e da massa seca da madeira, pois admite que a densidade da "substância madeira" é constante $(1,53 \mathrm{~g}$ $\mathrm{cm}^{-3}$ ) (Smith 1954; Foelkel et al. 1971). Comparaçōes feitas entre os dois métodos citados para a obtençáo da densidade básica mostraram não haver diferença significativa entre eles, não havendo influência também das dimensões do corpo de prova (Foelkel et al. 1971).

Há poucos trabalhos de pesquisa sobre densidade e teor de umidade de madeiras de espécies florestais nativas, exploradas comercialmente na Amazônia. Em alguns trabalhos são utilizados médias da densidade para a comunidade (Fearnside 1997; Nogueira 2008). Portanto, o presente trabalho tem como objetivo agrupar espécies nativas da floresta amazônica de terra firme a fim de compor cargas mistas para secagem artificial a partir dos atributos intrínsecos da madeira, densidade e teor de umidade.

\section{MATERIAL E MÉTODOS}

\section{Área de estudo}

Este estudo foi realizado em área de floresta amazônica de terra firme localizada no município de Itacoatiara, cerca de $227 \mathrm{~km}$ à leste de Manaus, AM. A área de estudo pertence à Empresa Mil Madeiras Preciosas Ltda. e é destinada a exploração de madeira para serraria, laminados e energia.

A temperatura média varia de 24 a $26^{\circ} \mathrm{C}$, com máxima de $38^{\circ} \mathrm{C}$ e mínima de $16^{\circ} \mathrm{C}$. A precipitação anual média é $2000 \mathrm{~mm}$. O clima da região é quente e úmido com três meses secos, classificado Amw segundo Köppen (Köppen 1948). A área tem relevo ondulado e o solo é classificado como latossolo amarelo distrófico de textura muito argilosa ou argilosa.

\section{Coleta de dados}

Para este estudo realizado em 20 ha de floresta amazônica de terra firme, foram selecionadas com base na representatividade em relação à biomassa de todas as espécies de interesse econômico registradas na área, nove espécies (Tabela 1). Explorando-se aproximadamente nove árvores por hectare com diâmetro mínimo de corte (DMC) de $50 \mathrm{~cm}$. Contudo,

Tabela 1 - Espécies e diâmetro a altura do peito (DAP) dos indivíduos selecionadas para o estudo de teor de umidade (\%) e densidade básica $\left(\mathrm{g} \mathrm{cm}^{-3}\right)$.

\begin{tabular}{|c|c|c|c|c|c|}
\hline \multirow{2}{*}{$\begin{array}{l}\text { Espécie } \\
\text { Brosimum parinarioides Ducke }\end{array}$} & \multirow{2}{*}{$\begin{array}{c}\text { Família } \\
\text { Moraceae }\end{array}$} & \multicolumn{4}{|c|}{$\begin{array}{l}\text { DAP dos indivíduos } \\
\text { selecionados (cm) }\end{array}$} \\
\hline & & 89 & 70 & 82 & \\
\hline Brosimum rubescens Taub. & Moraceae & 57 & 57 & 65 & \\
\hline Clarisia racemosa Ruiz \& Pav. & Moraceae & 54 & 66 & 58 & \\
\hline Lecythis poiteaui 0. Berg & Lecythidaceae & 61 & 73 & 70 & \\
\hline $\begin{array}{l}\text { Manilkara huberi (Ducke) } \\
\text { A. Chev. }\end{array}$ & Sapotaceae & 50 & 79 & 70 & \\
\hline $\begin{array}{l}\text { Mezilaurus itauba (Meisn.) } \\
\text { Taub. ex Mez }\end{array}$ & Lauraceae & 64 & 74 & 69 & \\
\hline Minquartia guianensis Aubl. & Olacaceae & 41 & 26 & 25 & \\
\hline Ocotea rubra Mez & Lauraceae & 121 & 92 & 71 & 61 \\
\hline Parkia paraensis Ducke & Leguminosae & 71 & 69 & 56 & \\
\hline
\end{tabular}

para a espécie Minquartia guianensis Aubl. considerou-se o DMC igual a $25 \mathrm{~cm}$, pois a madeira desta espécie é usada para produçáo de postes. 
As coletas foram realizadas na primeira quinzena de janeiro de 2011. Para cada espécie foram selecionados três indivíduos de diferentes diâmetros. Após o corte das árvores foram retiradas amostras em quatro posiçóes, tronco na base (TR-B), tronco no topo (TR-T), galhos grossos (GG) (com diâmetro maior que $10 \mathrm{~cm}$ ) e galhos finos (GF) (com diâmetro entre 2 e $10 \mathrm{~cm}$ ) (Higuchi et al. 1998).

O local de corte para derrubada foi considerado a base do tronco, o local imediatamente antes da primeira bifurcação foi considerado o topo. Para TR-B, TR-T, GG e GF foi retirado um disco de $5 \mathrm{~cm}$ de espessura. Para as duas primeiras posiçôes foi posteriormente retirada uma cunha por disco (aproximadamente um sexto do disco) e realizada a separação da casca e madeira. Para este estudo, de todas as amostras coletadas foram retiradas pequenas sub-amostras, com aproximadamente $1,5 \mathrm{~cm}$ de espessura. Todas as amostras foram coletadas uma semana após o corte da árvore.

Visando a determinaçáo do teor de umidade máximo de cada sub-amostra em cada posição, essas sub-amostras foram submersas em água para saturação até massa constante, foi realizada a pesagem da massa saturada (PF) (Barbosa e Ferreira 2004). As amostras foram então secas em estufa com circulação forçada de ar (MA-035/5 Marconi, Brasil) a 103 $\pm 2{ }^{\circ} \mathrm{C}$ até massa constante, determinando a massa seca (PS). A massa foi considerada constante quando a diferença entre duas pesagens consecutivas, com diferença de 24 horas, foi inferior a $1 \%$. O teor de umidade máximo (TU) foi calculado em relaçấo à base seca:

$$
T U(\%)=\frac{P F-P S}{P S}
$$

A densidade básica das amostras foi calculada pela fórmula proposta por Smith (1954), tendo como base o máximo teor de umidade.

$$
D b=\frac{1}{\frac{P F-P S}{P S}+\frac{1}{1,53}}
$$

Os valores referentes ao teor de umidade e densidade básica da madeira para as diferentes posiçôes e para as nove espécies foram submetidos à análise de variância com quatro tratamentos (TR T, TR B, GG, GF) e três repetiçóes. Foram verificadas as diferenças significativas, para teor de umidade e densidade básica, entre posição na árvore a partir do teste de Tukey a 5\% de significância. Para o agrupamento das espécies a partir da média das posiçóes TR T e TR B de cada indivíduo, foi utilizado o teste Scott-Knott a 5\% de significância, com nove espécies e três repetiçôes.

\section{RESULTADOS E DISCUSSÃO}

\section{Teor de umidade}

As madeiras ficaram na estufa com circulação forçada de ar por 12 dias. O teor de umidade máximo por espécie e por posiçáo pode ser observado na Tabela 2 . Na maioria das espécies os galhos apresentaram maiores teores de umidade ( $\mathrm{p}$ $<0,05)$, em relação ao tronco. Os galhos têm lenho mais jovem

Tabela 2 - Teores médios de umidade (\%) por espécie e por posição da árvore.

\begin{tabular}{lccccc}
\hline Espécie & GF & GG & TR-B & TR-T & $\begin{array}{c}\text { Média } \\
\text { TR }\end{array}$ \\
\hline Brosimum parinarioides & $88,1 \mathrm{a}$ & $112,7 \mathrm{~b}$ & $98,4 \mathrm{ab}$ & $111,6 \mathrm{a}$ & 105,0 \\
\hline Brosimum rubescens & $82,8 \mathrm{a}$ & $88,6 \mathrm{a}$ & $57,4 \mathrm{bd}$ & $52,0 \mathrm{~cd}$ & 54,7 \\
\hline Clarisia racemosa & $109,9 \mathrm{~b}$ & $107,2 \mathrm{~b}$ & $78,0 \mathrm{a}$ & $94,1 \mathrm{ab}$ & 86,0 \\
\hline Lecythis poiteaui & $81,1 \mathrm{a}$ & $67,3 \mathrm{~b}$ & $43,6 \mathrm{c}$ & $48,1 \mathrm{c}$ & 45,8 \\
Manilkara huberi & $71,1 \mathrm{~b}$ & $60,5 \mathrm{ab}$ & $49,2 \mathrm{a}$ & $48,6 \mathrm{a}$ & 48,9 \\
Mezilaurus itauba & $69,0 \mathrm{a}$ & $71,5 \mathrm{ac}$ & $44,3 \mathrm{bd}$ & $46,9 \mathrm{~cd}$ & 45,6 \\
Minquartia guianensis & $66,2 \mathrm{a}$ & $56,1 \mathrm{ac}$ & $45,5 \mathrm{a}$ & $55,2 \mathrm{a}$ & 50,4 \\
Ocotea rubra & $61,0 \mathrm{a}$ & $66,6 \mathrm{a}$ & $63,0 \mathrm{bc}$ & $60,4 \mathrm{ac}$ & 61,7 \\
\hline Parkia paraensis & $89,3 \mathrm{a}$ & $105,0 \mathrm{a}$ & $111,0 \mathrm{a}$ & $117,3 \mathrm{a}$ & 114,1 \\
\hline
\end{tabular}

GF: galho fino (diâmetro de 2 a $10 \mathrm{~cm}$ ), GG: galho grosso (diâmetro $>10 \mathrm{~cm}$ ), TR B: base do tronco, TR T: topo do tronco. Médias seguidas por uma mesma letra na linha, não diferem pelo teste Tukey $(p>0,05)$.

que o tronco, maior proporçáo de tecidos ativos e, portanto, maior proporção de água quando comparados ao tronco.

Os teores de umidade das espécies Minquartia guianensis e Parkia paraensis não diferiu significativamente entre as posiçôes $(\mathrm{p}>0,05)$. Apenas as espécies Brosimum parinarioides ( $\mathrm{p}<$ $0,05)$ e Lecythis poiteaui $(\mathrm{p}<0,01)$ apresentaram diferença significativa na umidade entre galho fino e galho grosso.

Os teores de umidade não diferiram significativamente entre a base e o topo do tronco para as nove espécies, a média do teor de umidade na base foi de $65 \%$ e no topo foi de $68 \%$. Isso indica estabilidade dimensional ao longo do tronco para o processo de secagem. Considerando que o ponto de saturaçáa de fibras médio é 30\% (Glass e Zelinka 2010), a quantidade de água livre percentual de cada espécie variou de 30\% a 84\%. As espécies mais leves apresentam maior quantidade de água livre e esta água pode ser retirada mais facilmente durante o processo de secagem. Com base no teor de umidade do tronco na base e no topo, as espécies foram separadas em três grupos (Tabela 3). 
Tabela 3 - Agrupamento das espécies de acordo com as diferenças estatísticas do teor de umidade (TU) e densidade básica (DB).

\begin{tabular}{lcc}
\hline & Média TU $(\%)$ & Média DB $\left(\mathrm{g} \mathrm{cm}^{-3}\right)$ \\
\hline Grupo 1 & $54,73 \mathrm{a}$ & $0,835 \mathrm{a}$ \\
Brosimum rubescens & $45,82 \mathrm{a}$ & $0,900 \mathrm{a}$ \\
Lecythis poiteaui & $48,89 \mathrm{a}$ & $0,878 \mathrm{a}$ \\
Manilkara huberi & $45,56 \mathrm{a}$ & $0,904 \mathrm{a}$ \\
\hline Mezilaurus itauba & $50,36 \mathrm{a}$ & $0,867 \mathrm{a}$ \\
\hline Minquartia guianensis & & \\
\hline Grupo 2 & $86,02 \mathrm{~b}$ & $0,665 \mathrm{~b}$ \\
Clarisia racemosa & $74,08 \mathrm{~b}$ & $0,720 \mathrm{~b}$ \\
\hline Ocotea rubra & & \\
\hline Grupo 3 & $105,00 \mathrm{c}$ & $0,588 \mathrm{c}$ \\
\hline Brosimum parinarioides & $114,13 \mathrm{c}$ & $0,561 \mathrm{c}$ \\
\hline Parkia paraensis & & \\
\hline
\end{tabular}

\section{Densidade básica}

A média das densidades por espécie e por posição na árvore pode ser observada na Tabela 4. Em média as densidades do tronco foram maiores que as densidades dos galhos, em razão da maior proporçáo de lenho jovem nos galhos. Das nove espécies amostradas, três apresentaram densidade que variou de $0,561 \mathrm{a} 0,720 \mathrm{~g} \mathrm{~cm}^{-3}$ podendo ser classificadas como madeira de média densidade. A densidade das outras seis espécies variou de 0,835 a $0,909 \mathrm{~g} \mathrm{~cm}^{-3}$ e podem ser classificadas como madeira pesada (Melo et al. 1990). Essas madeiras são usadas pela empresa principalmente para confecção de pisos, postes, estacas e para construção pesada em geral.

Tabela 4 - Densidade básica $\left(\mathrm{g} \mathrm{cm}^{-3}\right)$ por espécie e por posição da árvore.

\begin{tabular}{|c|c|c|c|c|c|}
\hline Espécie & GF & $\mathrm{GG}$ & TR-B & TR-T & Média TR \\
\hline $\begin{array}{l}\text { Brosimum } \\
\text { parinarioides }\end{array}$ & $0,657 \mathrm{a}$ & 0,564 a & $0,611 \mathrm{a}$ & 0,566 a & 0,588 \\
\hline $\begin{array}{l}\text { Brosimum } \\
\text { rubescens }\end{array}$ & $0,676 \mathrm{a}$ & 0,650 a & $0,816 b$ & $0,854 \quad b$ & 0,835 \\
\hline Clarisia racemosa & $0,571 \mathrm{~b}$ & $0,581 \quad b$ & $0,701 \mathrm{a}$ & $0,629 a b$ & 0,665 \\
\hline Lecythis poiteaui & $0,683 \mathrm{a}$ & $0,754 \quad b$ & $0,918 \mathrm{c}$ & 0,882 c & 0,900 \\
\hline Manilkara huberi & $0,735 \mathrm{~b}$ & $0,795 a b$ & $0,877 \mathrm{a}$ & 0,879 a & 0,878 \\
\hline Mezilaurus itauba & $0,756 \mathrm{a}$ & $0,735 \mathrm{ac}$ & $0,915 b$ & $0,893 \mathrm{bc}$ & 0,904 \\
\hline $\begin{array}{l}\text { Minquartia } \\
\text { guianensis }\end{array}$ & $0,761 \mathrm{a}$ & 0,839 a & $0,903 \mathrm{a}$ & $0,831 \quad a$ & 0,867 \\
\hline Ocotea rubra & $0,612 \mathrm{a}$ & 0,625 a & $0,726 b$ & $0,715 a b$ & 0,720 \\
\hline Parkia paraensis & $0,648 \mathrm{a}$ & $0,591 \quad a$ & $0,573 \mathrm{a}$ & 0,549 a & 0,561 \\
\hline
\end{tabular}

GF: galho fino (diâmetro de 2 a $10 \mathrm{~cm}$ ), GG: galho grosso (diâmetro $>10 \mathrm{~cm}$ ), TR B: base do tronco, TR T: topo do tronco. Médias seguidas por uma mesma letra na coluna, não diferem pelo teste Tukey $(p>0,05)$.
Ao comparar as densidades encontradas com os dados do Laboratório de Produtos Florestais/Serviço Florestal Brasileiro (LPF/SFB) (disponível em: www.ibama.gov.br/lpf/ madeira), as espécies $M$. guianensis, $M$. huberi, B. rubescens, $B$. parinarioides e $C$. racemosa apresentaram densidades semelhantes. Já para as espécies $M$. itauba, O. rubra e $P$. paraensis, as densidades encontradas neste trabalho diferiram das densidades encontradas pelo LPF/SFB. Essa diferença entre densidades dentro de uma mesma espécie deve-se à diferença de sítios, considerando principalmente a diferença na fertilidade dos solos (Baker et al. 2004; Muller-Landau 2004; Malhi 2006), já que os dados do LPF/SFB foram obtidos a partir de madeiras coletadas no Estado do Pará e os dados do presente trabalho foram coletados no Estado do Amazonas, Amazônia Central.

Não foram encontradas na literatura informaçôes sobre densidade para a espécie $L$. poiteaui. Outras espécies do gênero Lecythis apresentam alta densidade básica, maior que 0,720 $\mathrm{g} \mathrm{cm}^{-3}$ (Coradin et al. 2010). A espécie L. pisonis (castanhasapucaia) apresenta densidade básica $\left(0,840 \mathrm{~g} \mathrm{~cm}^{-3}\right)$ bem próxima àquela da espécie estudada $\left(0,900 \mathrm{~g} \mathrm{~cm}^{-3}\right)$, assim a espécie $L$ poiteaui pode substituir a madeira da castanhasapucaia em construçôes, embarcaçóes e móveis. Mais estudos sobre características físico-mecânicas da madeira da espécie $L$. poiteaui devem ser realizados.

Não foi encontrada diferença significativa das densidades básicas entre as posiçōes galho grosso, galho fino e tronco para as espécies Minquartia guianensis $(\mathrm{p}=0,28)$, Brosimum parinarioides $(\mathrm{p}=0,11)$ e Parkia paraensis $(\mathrm{p}=0,22)$. Esse resultado indica uma homogeneidade nas características de densidade e umidade, sugerindo a possibilidade da caracterização das propriedades físicas densidade e teor de umidade dessas espécies a partir da análise dos galhos.

Para as outras espécies houve diferença das densidades básicas entre algumas posiçôes, porém não houve diferença significativa entre a densidade básica do tronco na base e no topo, conferindo estabilidade dimensional a madeira (Tabela 4).

A partir da densidade básica, as espécies foram separadas em três grupos pelo teste Scott-Knott (Tabela 3). O primeiro grupo é classificado como madeira pesada, o segundo e o terceiro grupo são classificados como madeira de média densidade segundo Melo et al. (1990).

Por conseguinte, a separação desses grupos pode ser usada para o processo de secagem da madeira. A variedade de espécies exploradas na Amazônia não possibilita operacionalmente a secagem de uma espécie por câmara de secagem, havendo, portanto a necessidade de agrupar as espécies com características semelhantes. Várias características 
podem ser usadas para agrupar espécies, porém as variáveis mais fáceis de determinar e que mais influenciam a secagem da madeira são densidade básica e teor de umidade (Simpson e Baah 1989; Nascimento et al. 1997). Estimativas de agrupamento por densidade básica são razoáveis, pois esta variável se relaciona com os defeitos de secagem (Simpson e Verrill 1997).

As madeiras mais densas, pertencentes ao grupo 1, são mais difíceis de secar pois são mais susceptíveis a defeitos (Simpson e Baah 1989), por apresentarem menor teor de umidade. $\mathrm{O}$ processo de secagem dessas madeiras demandam menor tempo até atingir $30 \%$ de umidade e maior tempo entre 30 a $12 \%$ de umidade em relaçấo às outras espécies estudadas (Marques 2008).

As espécies Brosimum rubescens, Mezilaurus itauba e Manilkara huberi pertencentes ao grupo 1 apresentam secagem lenta, com tendência a defeitos. Já as espécies Brosimum parinarioides e Clarisia racemosa pertencentes aos grupos 2 e 3 apresentam secagem rápida com tendência grande e moderada à defeitos respectivamente (IBDF/LPF 1981; IBDF/LPF 1988; Marques 2008).

\section{CONCLUSÕES}

As espécies Minquartia guianensis, Brosimum parinarioides, Clarisia racemosa, Lecythis poiteaui, Ocotea rubra, Mezilaurus itauba, Manilkara huberi, Brosimum rubescens e Parkia paraensis apresentam densidade média à alta, apresentando densidade básica da madeira na base do tronco semelhante do topo do tronco, sugerindo estabilidade mecânica da madeira. Essas espécies são utilizadas principalmente para serraria, laminados e postes, necessitando de estabilidade ao longo do tronco.

As nove espécies estudadas foram separadas em três grupos, e cada grupo pode compor cargas mistas para secagem em câmaras industriais. O primeiro grupo com as espécies Minquartia guianensis, Lecythis poiteaui, Mezilaurus itauba, Manilkara huberi, Brosimum rubescens apresentam maior densidade básica, necessitando maiores cuidados no processo de secagem para evitar defeitos na madeira.

\section{AGRADECIMENTOS}

Os autores agradecem ao projeto Instituto Nacional de Ciência e Tecnologia INCT - Madeiras da Amazônia e à Coordenaçáo de Aperfeiçoamento de Pessoal de Nível Superior (CAPES) pelo apoio financeiro, aos funcionários do Instituto Nacional de Pesquisas da Amazônia (INPA) Tertuliano Wesen, Antônio Nascimento e José Maria Gonçalves, pela colaboração no trabalho de campo.

\section{REFERÊNCIAS BIBLIOGRÁFICAS}

Andrade, A.; Jankowsky, I.P.; Ducatti, M.A. 2001. Grupamento de madeiras para secagem convencional. Scientia Forestalis, 59: 89-99.

Baker, T.R.; Phillips, O.L.; Malhi, Y.; Almeida, S.; Arroyo, L.; Fiore, A.; Erwin, T.; Killen, T.J.; Laurance, S.G.; Laurance, W.F.; Lewis, S.L.; Lloyd, J. 2004. Variation in wood density determines spatial patterns in amazonian forest biomass. Global Change Biology, 10: 545-562.

Barbosa, R.I.; Ferreira, C.A.C. 2004. Densidade básica da madeira de um ecossistema de "campina" em Roraima, Amazônia Brasileira. Acta Amazonica, 34(4): 587-591.

Coradin, V.T.R.; Camargos, J.A.A.; Pastore, T.C.M.; Christo, A.G. 2010. Madeiras comerciais do Brasil: chave interativa de identificação baseada em caracteres gerais e macroscópicos. (http://www.ibama.gov.br/lpf/madeira/introducao.htm). Acesso em 05/08/2011.

Fearnside, P.M. 1997. Wood density for estimating forest biomass in Brazilian Amazonia. Forest Ecology and Management, 90 (1): 59-87.

Foelkel, C.E.B.; Brasil, M.A.M.; Barrichelo, L.E.G. 1971. Métodos para determinaçáo da densidade básica de cavacos para coníferas e folhosas. Instituto de Pesquisas e Estudos Florestais, 2/3: 67-74.

Glass, S.V.; Zelinka, A.L. 2010. Moisture relations and physical properties of wood. General Technical Report FPL- GTR. 190: 20 pp.

Higuchi, N.; Santos, J.; Ribeiro, R.J.; Minette, L.; Biot, Y. 1998. Biomassa da parte aérea da vegetação da floresta tropical úmida de terra-firme da Amazônia brasileira. Acta Amazonica, 28 (2): 153-166.

Instituto Brasileiro de Desenvolvimento Florestal/Laboratório de Produtos Florestais (IBDF/LPF). 1988. Madeiras da Amazônia, caracteristicas e utilização; Estação experimental de Curuá-Una. Brasília. 236 pp.

Instituto Brasileiro de Desenvolvimento Florestal/Laboratório de Produtos Florestais (IBDF/LPF). 1981. Madeiras da Amazônia: características e utilização. 1. Floresta Nacional do Tapajós, CNPq: Brasília. 156 pp.

Köppen, W. 1948. Climatologia: con un estudio de los climas de la tierra. Fondo de Cultura Econômica. México. 479 pp.

Logsdon, N.B.; Calil Junior, C. 2002. Influência da umidade nas propriedades de resistência e rigidez da madeira. Cadernos de Engenharia de Estruturas, 18: 77-107.

Malhi, Y.; Wood, D.; Baker, T.R.; Wright, J.; Phillips, O.L.; Cochrane, T.; Meir, P.; Chave, J.; Almeida, S.; Arroyo, L.; Higuchi, N. 2006. The regional variation of aboveground live biomass in old-growth Amazonian forests. Global Change Biology, 12: $1107-1138$

Marques, M.H.B. 2008. Agrupamento de 41 espécies de madeiras da amazônia para secagem baseado em características anatômicas e físicas. Doutorado, Universidade de Brasília, Brasília. 141 pp. 
Melo, J.E.; Coradin, V.T.R.; Mendes, J.C. 1990. Classes de densidade de madeira para a Amazônia brasileira. In: Anais do Congresso Florestal Brasileiro 6: 695-699. São Paulo, SP, Brasil.

Muller-Landau, H.C. 2004. Interspecific and inter-site variation in wood specific gravity of tropical trees. Biotropica, 36 (1): 20-32.

Nascimento, C.C.; Garcia, J.N.; Diáz, M.P. 1997. Agrupamento de espécies madeireiras da amazônia em função da densidade básica e propriedades mecânicas. Madera y Bosques, 3 (1): 33-52.

Nogueira, E.M.; Fearnside, P.M.; Nelson, B.W. 2008. Normalization of wood density in biomass estimates of Amazon forests. Forest Ecology and Management, 256: 990-996.
Simpson, W.T.; Baah, C.K. 1989. Grouping tropical wood species for kiln drying. Madison, WI: U.S. Dept. of Agriculture, Forest Service, Forest Products Laboratory. 14 pp.

Simpson, W.T.; Verrill, S.P. 1997. Estimating kiln schedules for tropical and temperate hardwoods using specific gravity. Forest Products Journal, 47 (7/8): 64-68.

Smith, D.M. 1954. Maximum moisture content method for determining specific gravity of small wood samples. USDA Forest Products Laboratory No 2014.8 pp.

Recebido em: 23/10/2011

Aceito em: 31/05/2012 